\title{
Article \\ Extracellular Vesicles Allow Epigenetic Mechanotransduction between Chondrocytes and Osteoblasts
}

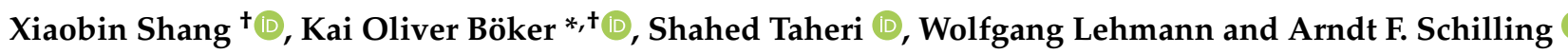 \\ Department of Trauma Surgery, Orthopedics and Plastic Surgery, University Medical Center Göttingen, \\ 37075 Göttingen, Germany; xiaobin.shang@stud.uni-goettingen.de (X.S.); \\ shahed.taheri@med.uni-goettingen.de (S.T.); Wolfgang.Lehmann@med.uni-goettingen.de (W.L.); \\ Arndt.schilling@med.uni-goettingen.de (A.F.S.) \\ * Correspondence: kai.boeker@med.uni-goettingen.de \\ † These authors contributed equally to this work.
}

Citation: Shang, X.; Böker, K.O.;

Taheri, S.; Lehmann, W.; Schilling, A.F. Extracellular Vesicles Allow Epigenetic Mechanotransduction between Chondrocytes and Osteoblasts. Int. J. Mol. Sci. 2021, 22, 13282. https://doi.org/10.3390/ ijms222413282

Academic Editor: Gabriela Loots

Received: 12 November 2021

Accepted: 7 December 2021

Published: 10 December 2021

Publisher's Note: MDPI stays neutral with regard to jurisdictional claims in published maps and institutional affiliations.

Copyright: (c) 2021 by the authors. Licensee MDPI, Basel, Switzerland. This article is an open access article distributed under the terms and conditions of the Creative Commons Attribution (CC BY) license (https:// creativecommons.org/licenses/by/ $4.0 /)$.

\begin{abstract}
MicroRNAs (miRNAs) can be transported in extracellular vesicles (EVs) and are qualified as possible messengers for cell-cell communication. In the context of osteoarthritis (OA), miR-221-3p has been shown to have a mechanosensitive and a paracrine function inside cartilage. However, the question remains if EVs with miR-221-3p can act as molecular mechanotransducers between cells of different tissues. Here, we studied the effect of EV-mediated transport in the communication between chondrocytes and osteoblasts in vitro in a rat model. In silico analysis (Targetscan, miRWalk, miRDB) revealed putative targets of miRNA-221-3p (CDKN1B/p27, TIMP-3, Tcf712/TCF4, ARNT). Indeed, transfection of miRNA-221-3p in chondrocytes and osteoblasts resulted in regulation of these targets. Coculture experiments of transfected chondrocytes with untransfected osteoblasts not only showed regulation of these target genes in osteoblasts but also inhibition of their bone formation capacity. Direct treatment with chondrocyte-derived EVs validated that chondrocyte-produced extracellular miR-221-3p was responsible for this effect. Altogether, our study provides a novel perspective on a possible communication pathway of a mechanically induced epigenetic signal through EVs. This may be important for processes at the interface of bone and cartilage, such as OA development, physiologic joint homeostasis, growth or fracture healing, as well as for other tissue interfaces with differing biomechanical properties.
\end{abstract}

Keywords: osteoarthritis; microRNA; extracellular vesicles; cell-cell communication; mechanical loading

\section{Introduction}

Osteoarthritis (OA) is a disease of the whole joint characterized by chronic inflammation, cartilage degradation, and subchondral bone remodeling, which can lead to severe pain and disability in patients [1-3]. It is estimated that more than 600 million people over the age of 40 years are living with symptomatic knee OA, resulting in individual suffering and substantial socioeconomic costs [4-6]. Current treatment of OA focuses on pain therapy and inflammation treatment and the only option for endpoint OA patients is total knee arthroplasty surgery [7]. So far, no disease-modifying treatments are available, despite decades of research. Therefore, further study on physiopathologic mechanisms of joint $\mathrm{OA}$ is urgent to explore pioneering therapeutic regimens.

As nano-size vesicles wrapped by a phospholipid bilayer, Extracellular Vesicles (EVs) derived from chondrocytes could promote chondrocyte proliferation and migration in a paracrine fashion [8]. MicroRNAs (miRNAs), which can be transported in EVs, have been demonstrated to participate in OA pathophysiology through post-transcriptional regulation of target mRNAs [9-12]. In particular, miR-221 has been shown to be regulated in arthritic cartilage and the expression of miR-221 is upregulated under mechanical loading [13-16]. The joint is exposed to dynamic mechanical loading [17] and mechanics 
play a key role in the regulation of joint function and dysfunction. This leads to adaptive changes in cartilage and subchondral bone [18]. Due to its biomechanical properties, at the same load, cartilage is much more deformed than bone [18], making mechano-modulation of chondrocytes (e.g., via autocrine and paracrine signaling) a probable mechanism for the maintenance of joint homeostasis [19]. As in the joint, the subchondral bone is in direct contact with cartilage, and microchannels between the tissues theoretically allow cartilagebone crosstalk [20-24]. The question remains if EVs can also modulate cells of the adjacent tissue, thereby translating the mechanical information into a tissue-border-transcending epigenetic signal.

In this study, we investigated the role of EV-mediated transport of miRNA-221-3p in the communication between chondrocytes and osteoblasts in vitro. We hypothesized that the dynamic expression of miRNA-221-3p in cartilage chondrocytes may affect the molecular activity and the bone formation capacity of osteoblasts through EVs.

\section{Results}

\subsection{Identification of Chondrocyte Secreted EVs}

EVs were successfully isolated from the conditioned supernatant of chondrocytes by ultracentrifugation. Nanoparticle tracking analysis (NTA) demonstrated that the size distribution of EVs ranged from 50 to $200 \mathrm{~nm}$ (Figure 1a,b). Transmission electron microscopy (TEM) further showed a typical cup-or sphere-shaped-morphology of EVs with a bilayer membrane structure (Figure 1c). Further analysis demonstrated that transfection of miR-221-3p mimics did not affect the average size and amount of EVs compared to control miRNA transfections (Figure 1d,e). Characteristic markers such as CD81, Alix, and TSG101 proteins were positively expressed in EVs based on Western blot analysis (Figures $1 \mathrm{f}$ and S4).

$\mathbf{a}$

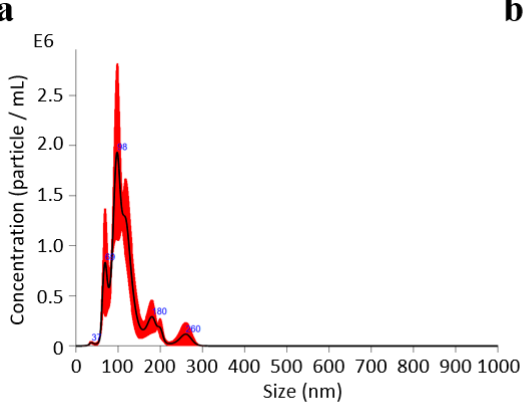

d

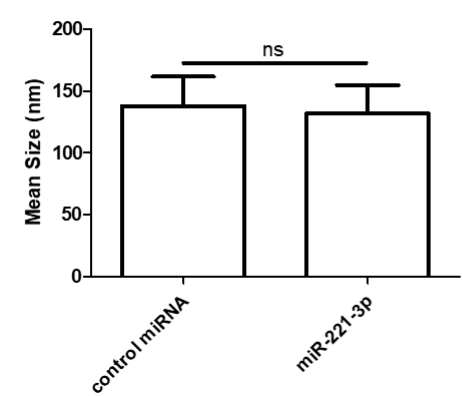

b

e
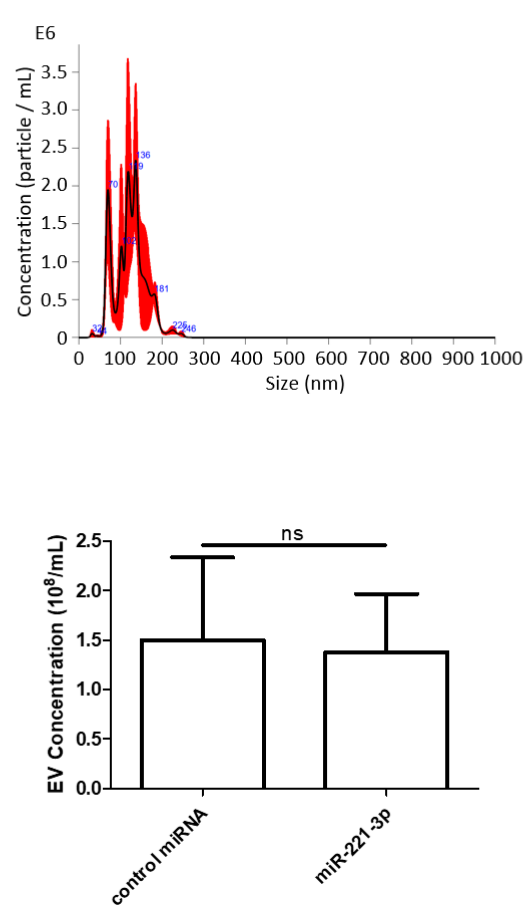

c

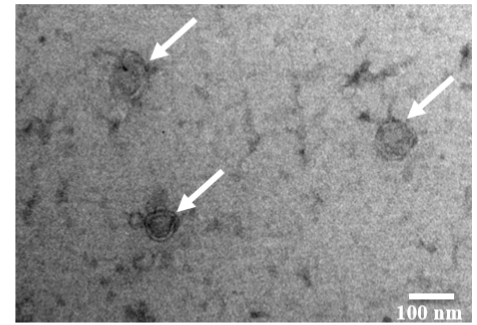

f

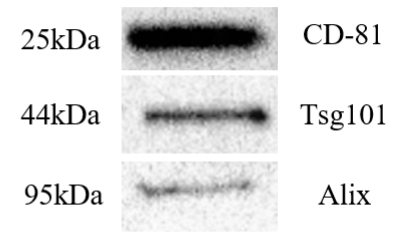

Figure 1. EVs were isolated by ultracentrifugation and identified by NTA, TEM and Western blotting. Particle size distribution of scramble miRNA-loaded (a) and miRNA-221-3p-loaded EVs (b) isolated by ultracentrifugation was determined by nanoparticle tracking analysis (NTA). Typical morphology (white arrows) of EVs was observed under TEM (c) scale bar $100 \mathrm{~nm}$. Transfection of miRNA-221-3p did not affect average size (d) and concentration (e) of EVs compared to control RNA transfections. Exosome-specific protein markers CD81, TSG101 and Alix were detected by Western blotting (f). ns stands for not statistically significant. 


\subsection{MiR-221-3p Expression in $O A$}

We successfully constructed an in vitro OA model with IL-1 $\beta$-treated chondrocytes. Details about the expression levels of OA-related genes can be found in supplementary data. In brief, the expression level of catabolic genes MMP-13, ADAMTS-5, and COX2 in IL$1 \beta$-induced chondrocytes were significantly increased while the anabolic gene SOX-9 was decreased (Figure S3a-d). Further analysis showed significantly downregulated expression of miR-221-3p in IL-1 $\beta$-treated chondrocytes versus control (Figure S3e).

\subsection{Mir-221-3p and Target Gene Analysis in Chondrocytes and Osteoblasts}

To determine the effect of miR-221-3p on chondrocytes, chondrocytes were transfected with miR-221-3p mimic and scrambled control. As shown in Figure 2a, the miR-221-3p expression was increased by $\sim 200$ fold $(p<0.001)$ after $48 \mathrm{~h}$ of transfection.

$\mathbf{a}$

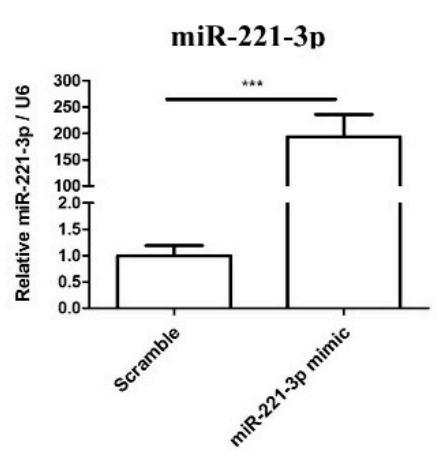

d

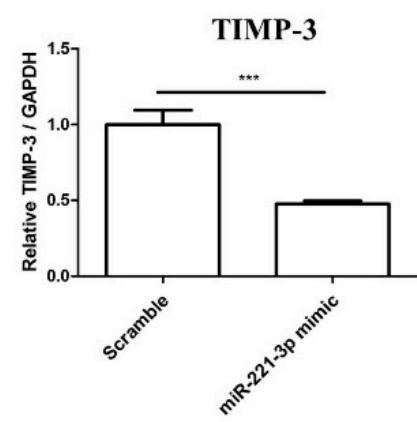

b

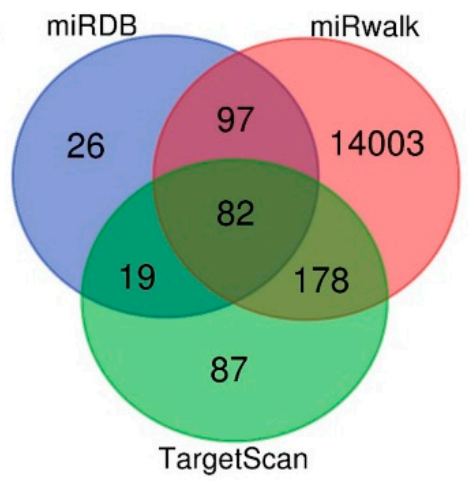

e

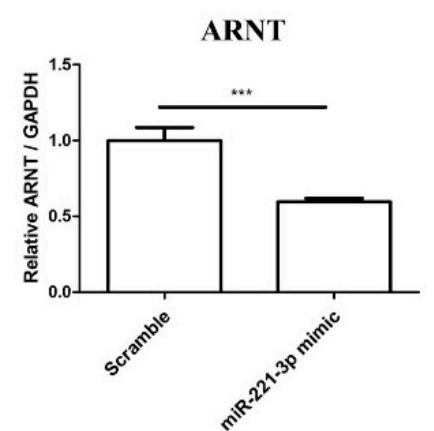

c

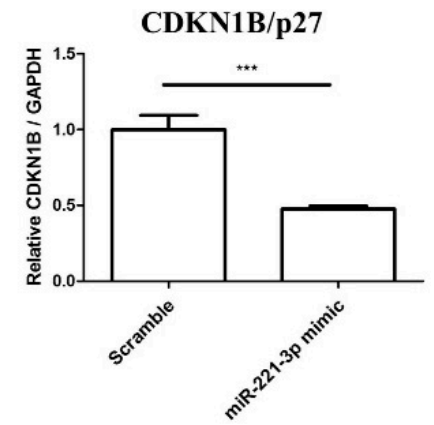

f Tef712/TCF4

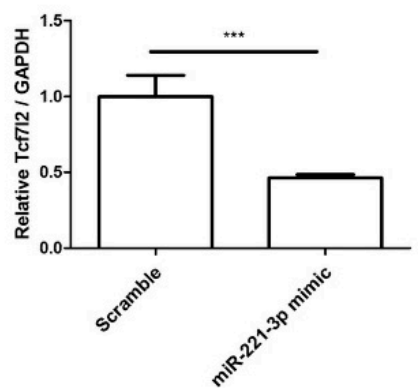

Figure 2. MiR-221-3p was increased by mimic transfection and the targets were predicted and identified in chondrocytes. (a) The transfection efficiency of the miR-221-3p mimic was confirmed by RT-qPCR relative to U6. (b) Predicted targets of miR-221-3p were identified using three independent platforms, i.e., miRDB, TargetScan, and miRwalk. (c-f) Chondrocytes were transfected with miR-scramble and miR-221-3p mimic, while the expression level of predicted targets was measured relative to GAPDH by RT-qPCR and ${ }^{* * *} p<0.001$ vs. corresponding control.

Thereafter, we predicted downstream target mRNAs using the publicly available websites miRDB, miRwalk, and TargetScan (Figure 2b). Given the potential role of miR-221$3 p$ in chondrocytes and osteoblasts homeostasis as reported in the literature, we chose four possible targets including Cyclin-dependent kinase inhibitor 1B (CDKN1B/p27), Tissue inhibitor of metalloproteinase 3 (TIMP-3), Transcription factor 7-like 2 (Tcf7l2/TCF4), and aryl hydrocarbon receptor nuclear translocator (ARNT) for further research. Indeed, all of these four genes were identified as direct targets of miR-221-3p by qRT-PCR (Figure 2c-f).

To understand the potential role of miR-221-3p in osteoblast differentiation, osteoblasts were transfected with miR-221-3p mimic or scrambled control. Osteogenic differentiation was evaluated by histology and gene expression analysis. The miR-221-3p expression was increased in treated osteoblasts by $\sim 2725$ fold $(p<0.001)$ (Figure 3a). MiR-221-3p overexpression inhibited the expression of the aforementioned targets CDKN1B/p27 (85\%, 
$p<0.001)$, ARNT (54\%, $p<0.001)$, TIMP-3 (75\%, $p<0.001)$ and Tcf7l2/TCF4 $(60 \%, p<0.001)$ (Figure 3b-e). More importantly, compared with the scrambled control, osteogenic markers such as COL1A1, RUNX2, and OCN were inhibited by $26 \%(p<0.001), 74 \%(p<0.001)$, and $97 \%(p<0.001)$ (Figure $3 \mathrm{f}-\mathrm{h}$ ), suggesting an influence of miR-221-3p on osteoblastic function. Indeed, Von Kossa and Alizarin Red staining also showed an apparent reduction in bone formation potential by miR-221-3p versus scrambled control (Figure 4).

$\mathbf{a}$

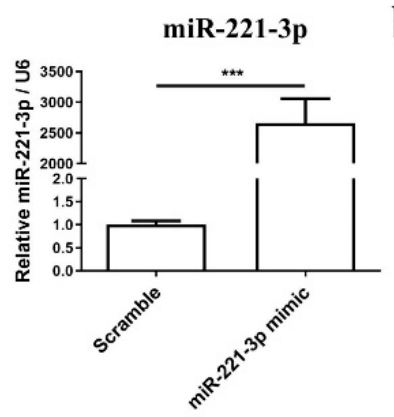

e

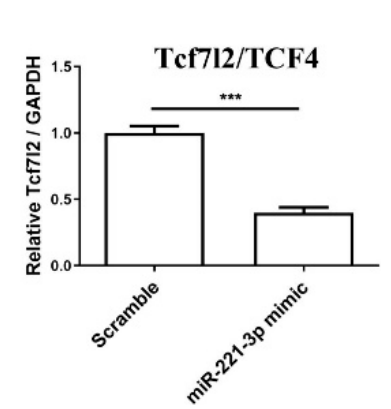

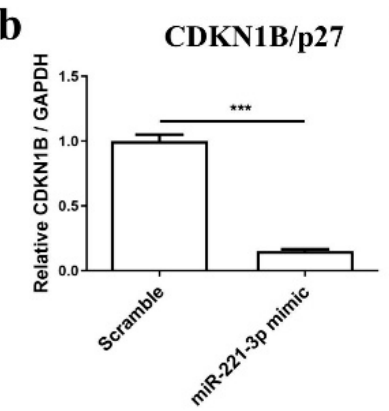

f

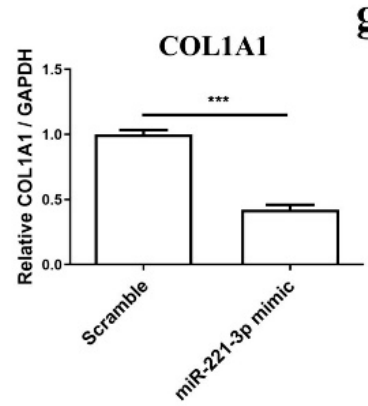

c

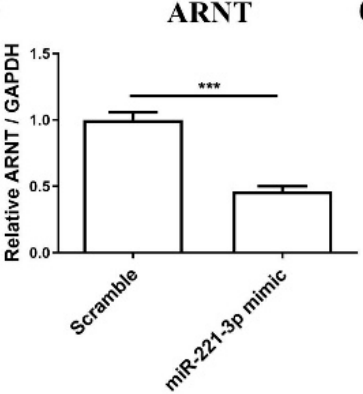

g

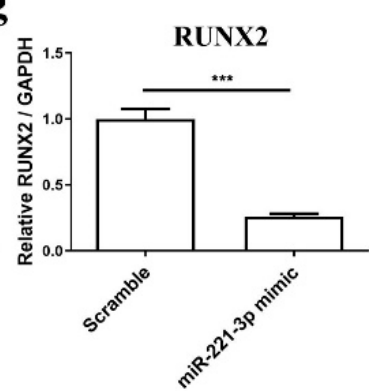

d

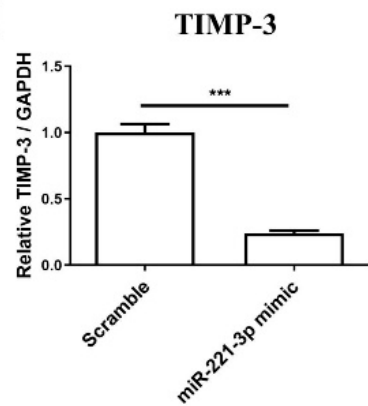

h

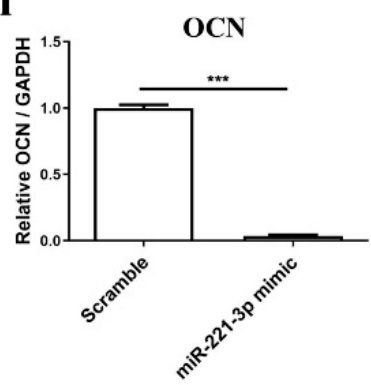

Figure 3. MiR-221-3p can act in osteoblasts. Osteoblasts were transfected with miR-scrambled or miR-221-3p mimic for 2 weeks in ODM, the expression level of miR-221-3p (a), the expression of putative targets of miR-221-3p, CDKN1B/p27 (b), ARNT (c), TIMP-3 (d), and Tcf712/TCF4 (e) and osteogenic markers COL1A1 (f), RUNX2 (g), OCN (h) were measured by RT-qPCR. CDKN1B/p27, ARNT, TIMP-3, Tcf712/TCF4, COL1A1, RUNX2, OCN relative to GAPDH, and miR-221-3p relative U6. ${ }^{* * *} p<0.001$ vs. corresponding control.

$\mathbf{a}$

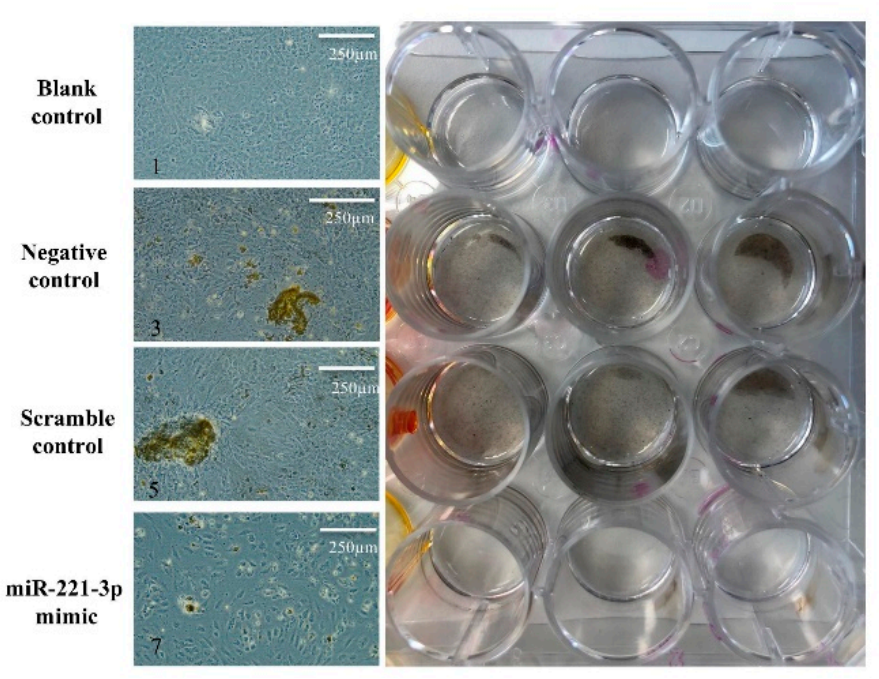

b

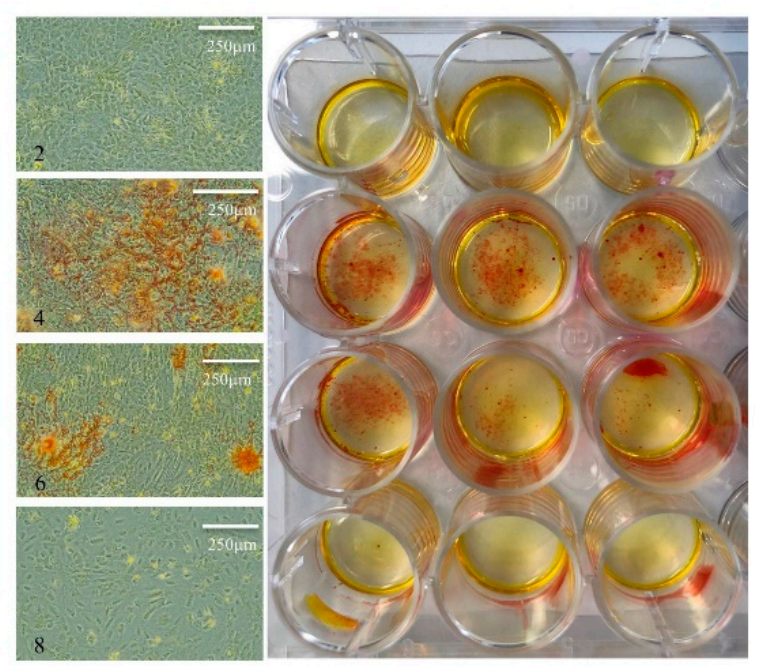

Figure 4. MiR-221-3p decreases osteoblast mineralization. The microscopic and macroscopic results of Von Kossa staining (a) and Alizarin Red staining (b) for osteoblasts after 2 weeks' culture in different conditions. Negative control without miRNA treatment $(3,4)$, scramble control $(5,6)$ and miR-221-3p mimic $(7,8)$ were cultured with ODM. The undifferentiated blank control group $(1,2)$ was cultured with BM. BM = Basal medium, ODM = Osteogenic differentiation medium. 
Next, we established an in vitro co-culture model to research chondrocyte-osteoblast communication. As shown in (Figure 5a), chondrocytes were transfected with miR-221-3p mimic or scrambled control and seeded in the lower transwell chamber, as a molecular simulation of a mechanically challenged or resting cartilage layer. Then, untreated osteoblasts were seeded in the upper chamber, representing the subchondral bone osteoblasts.

$\mathbf{a}$

Schematic diagram of co-culture

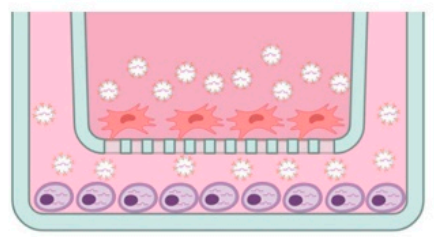

b

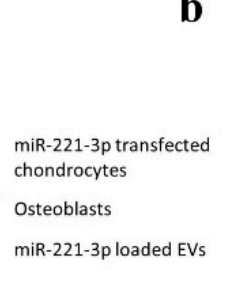

miR-221-3p loaded EVs

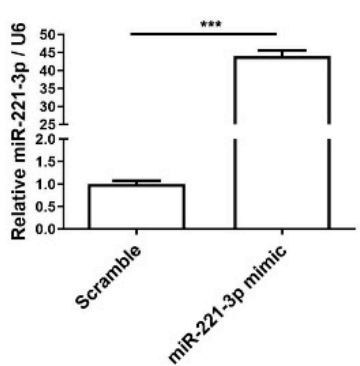

c

\section{EVs treated osteoblasts}

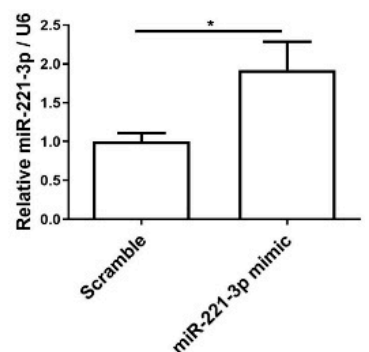

Figure 5. Chondrocyte signals through miR-221-3p can be transferred to osteoblasts via extracellular vesicles (a). Osteoblasts and chondrocytes were cocultured in a transwell system. miR-221-3p was overexpressed in EVs secreted by transfected chondrocytes (b). miR-221-3p in osteoblasts was significantly increased when cocultured with transfected chondrocytes (c). miRNA expression relative to U6. ${ }^{*} p<0.05$ and ${ }^{* * *} p<0.001$ vs. corresponding control. This figure was partly created with BioRender.com (May 2021).

After $48 \mathrm{~h}$ of coculture, the miR-221-3p expression in EVs isolated from the coculture medium was markedly increased by $\sim 45$ fold $(p<0.001)$ (Figure $5 b)$. The qRT-PCR analysis also indicated that miR-221-3p was expressed in the scrambled control EVs with a $\sim 26 \mathrm{ct}$ value in $10 \mu \mathrm{g}$ total extracellular RNA. This value increased to $20 \mathrm{ct}$ upon miR-221-3p mimic transfection of the chondrocytes (Figure S3h-i). This result revealed that the cargo of EVs contained the signal of miR-221-3p and that the expression of miR-221-3p in EVs could be increased by transfection of the maternal cells. Meanwhile, miR-221-3p expression in osteoblasts was significantly increased in the miR-221-3p mimic group versus scrambled control (Figure 5c).

Further results demonstrated that direct target genes of miR-221-3p including CDKN1B $/$ p27 (53\%, $p<0.001)$, ARNT (34\%, $p<0.001)$, TIMP-3 (51\%, $p<0.001)$ and Tcf712/TCF4 (52\%, $p<0.001)$ were significantly downregulated and osteogenic markers including COL1A1 and RUNX2 were inhibited by $~ 40 \%(p<0.001)$ and $32 \%(p<0.01)$ (Figure $6 a-g)$.

\subsection{Effect of Mir-221-3p Loaded EVs on Osteoblasts}

To clarify, if the observed effect was indeed dependent on EV-transport of miR-221-3p from chondrocytes to osteoblasts, we isolated EVs from the supernatant of chondrocytes transfected with miR-221-3p mimic or scrambled control, then treated the osteoblasts with EVs directly.

Firstly, $4.0 \times 10^{4}$ osteoblasts were treated with $5.0 \times 10^{8}$ miR-221-3p-loaded EVs or scramble control-loaded EVs for $48 \mathrm{~h}$. The number of chondrocytes used for EV isolation and the number of EV-treated osteoblasts was calculated with a ratio of 6:1, which was the same as in the coculture experiment. The results demonstrated a significant inhibitory effect of the same targets of miR-221-3p and osteogenic markers (Figure 7a-g). Briefly, the targets including CDKN1B/p27, ARNT, TIMP-3, and Tcf712/TCF4 were inhibited by 33\% $(p<0.001), 30 \%(p<0.001), 32 \%(p<0.001)$ and $37 \%(p<0.001)$, and osteogenic markers including COL1A1 and RUNX2 were inhibited by $~ 33 \%(p<0.001)$ and $21 \%(p<0.01)$, respectively. 

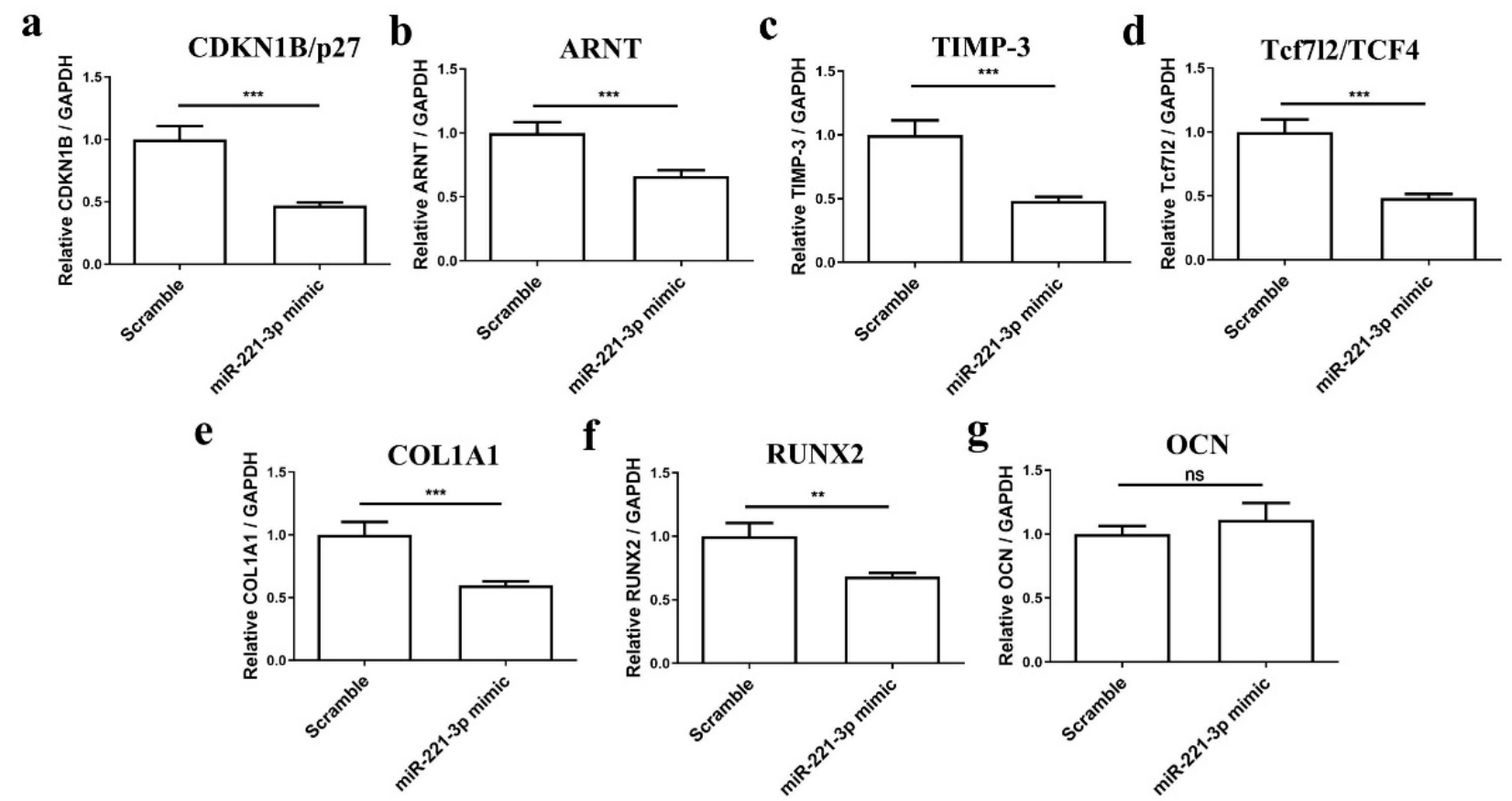

Figure 6. Secreted molecules from chondrocytes inhibited targets expression of miR-221-3p and osteogenic markers in osteoblasts. CDKN1B/p27 (a), ARNT (b), TIMP-3 (c), and Tcf7l2/TCF4 (d), and osteogenic markers (e-g), COL1A1, RUNX2, and OCN in osteoblasts cocultured with chondrocytes transfected with miR-221-3p mimic compared with scrambled control. Relative to GAPDH. ns stands for not statistically significant, ${ }^{* *} p<0.01$, and ${ }^{* * *} p<0.001$ vs. corresponding control.
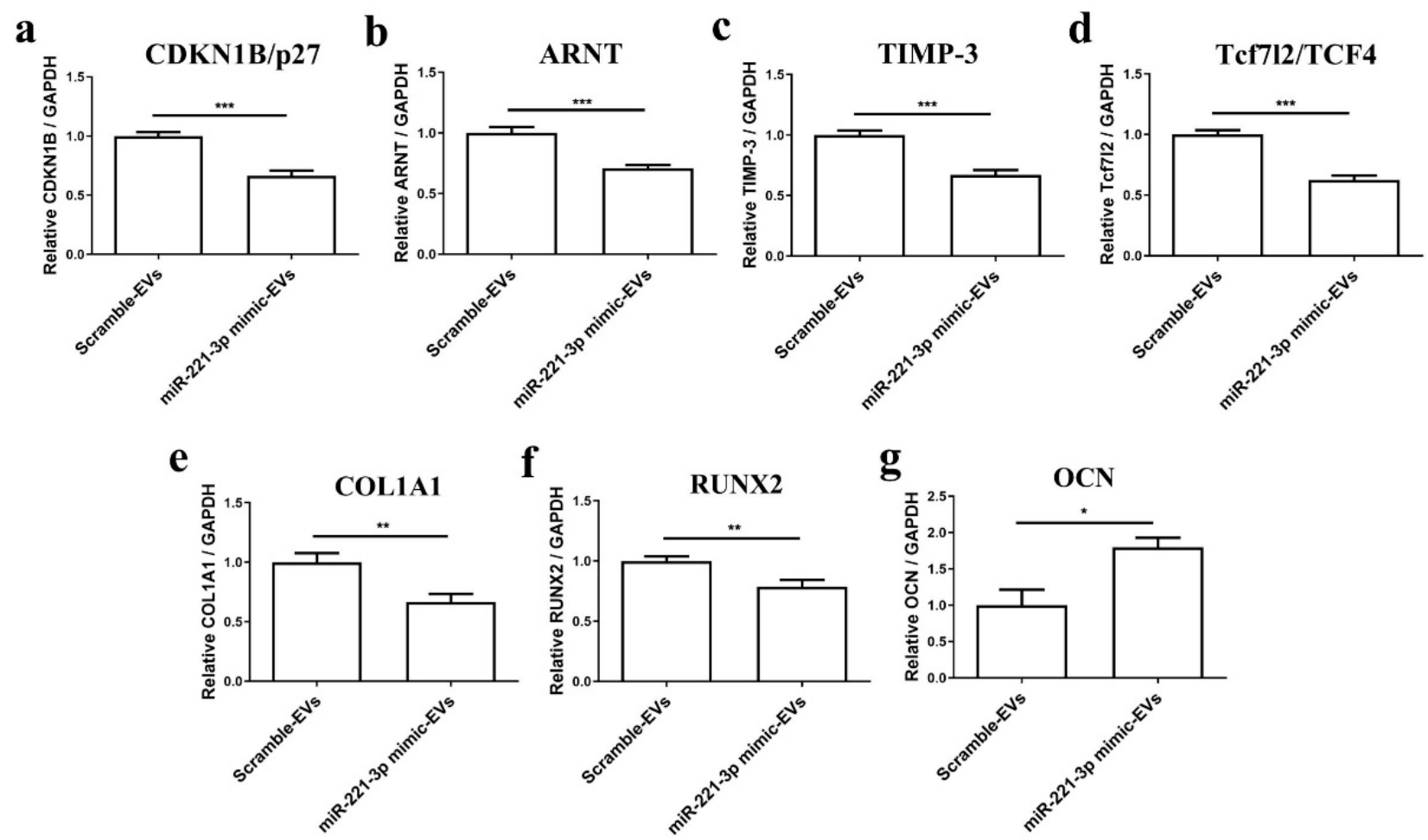

Figure 7. Chondrocytes secreted miR-221-3p-EVs inhibited osteogenic markers and targets expression of miR-221-3p in osteoblasts after $48 \mathrm{~h}$ coculture. The expression level of and targets expression (a-d) of miR-221-3p CDKN1B/p27, ARNT, TIMP-3, and Tcf712/TCF4, and osteogenic markers (e-g), COL1A1, RUNX2, and OCN in osteoblasts cocultured with EVs modified by mir-221-3p mimic compared with miRNA scramble after $48 \mathrm{~h}$. Relative to GAPDH., ${ }^{*} p<0.05,{ }^{* *} p<0.01$ and *** $p<0.001$ vs. corresponding control. 
Thereafter, osteoblasts were treated with miR-221-3p-loaded EVs or scramble control EVs in osteogenic differentiation medium (ODM) for 2 weeks according to the same protocol as above. The cells demonstrated obvious suppressed capacity for mineralized nodule formation verified by Alizarin Red staining (Figure 8a,b) and quantification (Figure 8c). After osteoblasts treatment with miR-221-3p-loaded EVs in ODM for 2 weeks, the mRNA expression of the osteoblastic markers COL1A1, RUNX2, and OCN was measured by qRT-PCR (Figure $8 \mathrm{~d}-\mathrm{f}$ ). The results showed a significant reduction in OCN expression $(\sim 86 \% p<0.001)$, while no significant difference was observed in COL1A1 and RUNX2. This indicated an inhibition of the osteogenic capacity of osteoblasts by the miR-221-3ploaded EVs.

a

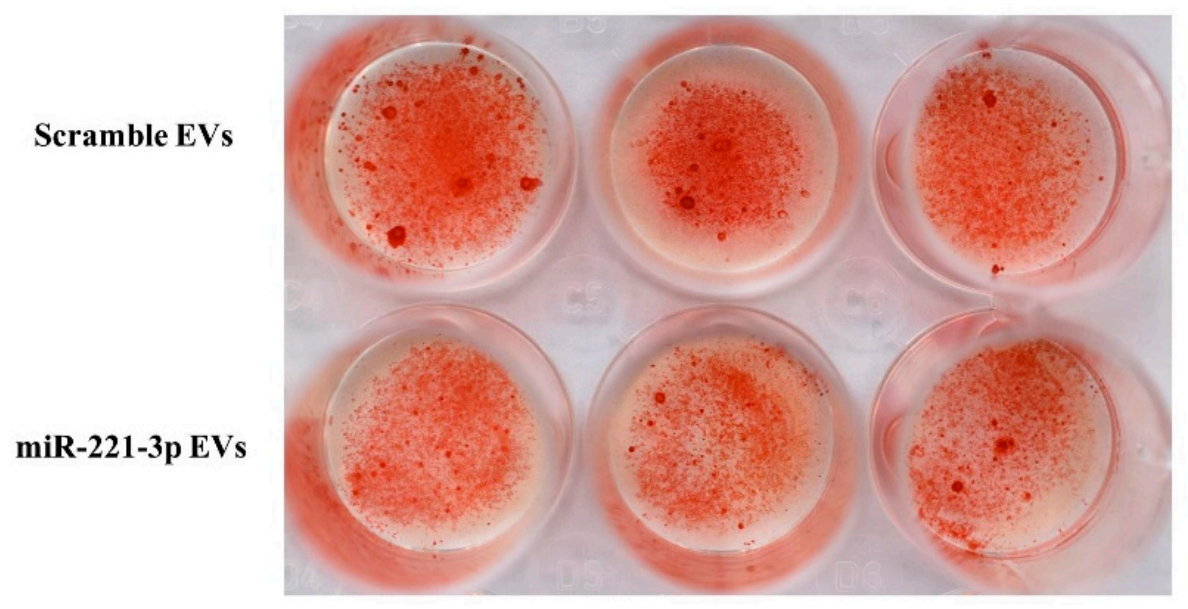

\section{b Alizarin Red Staining}
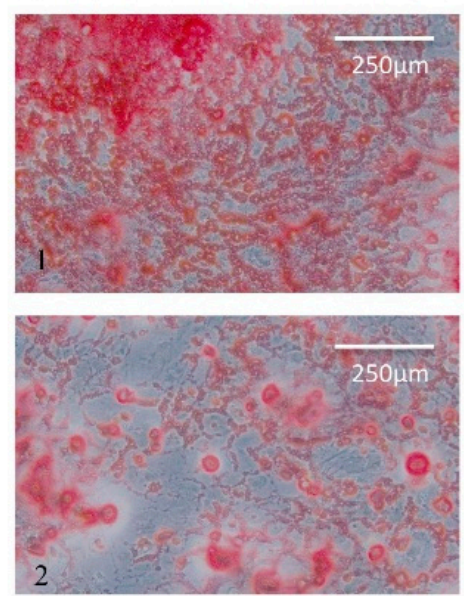
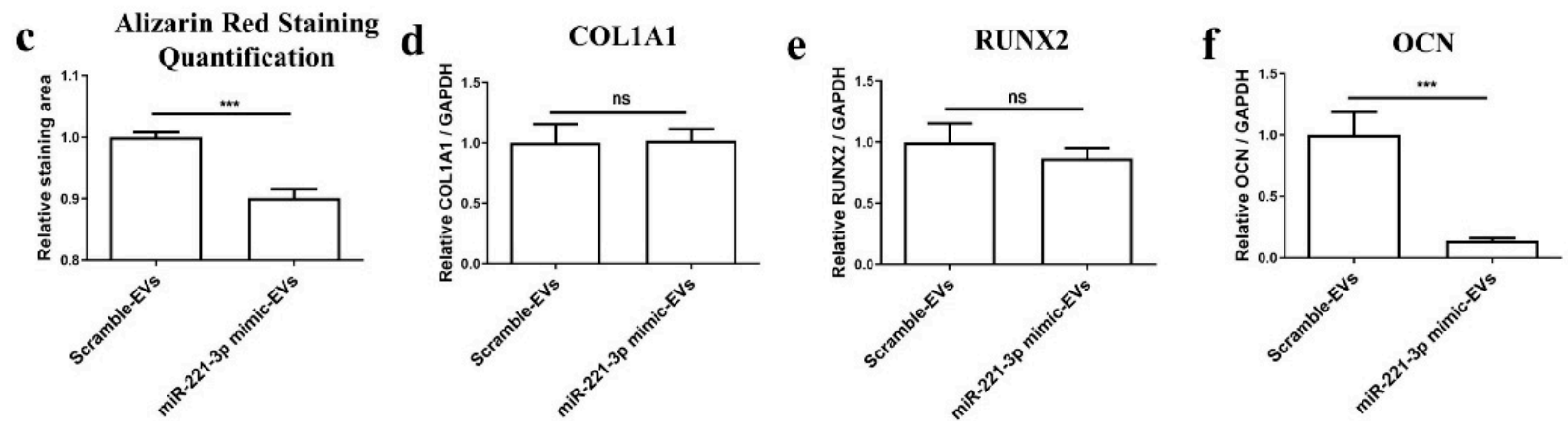

Figure 8. Osteoblasts were treated with EVs isolated from chondrocytes supernatant with or without miR-221-3p overexpression for 2 weeks in ODM. The microscopic (a) and macroscopic (b) result of Alizarin Red staining, respectively. Quantitative analysis of the Alizarin Red staining area through ImageJ (c), threshold $=120$. The relative expression level of osteogenic markers (d-f), COL1A1, RUNX2, and OCN in the two groups. Relative to GAPDH. ns stands for not statistically significant, ${ }^{* * *} p<0.001$ vs. corresponding control. ODM $=$ Osteogenic differentiation medium.

Collectively, our results revealed that miR-221-3p can be transferred from chondrocytes to osteoblasts via EVs and that miR-221-3p-loaded EVs derived from chondrocytes can inhibit osteoblastic function in vitro. However, this inhibitory effect may also be mediated by another source: EVs' parental cells modified by the transfection may also secrete EVs that are different not only by their miRNAs' content, but also by the hundreds of miRNAs' targets, which can also be modified during the transfection of the parental cells and the biogenesis of EVs. Furthermore, the presence of the miRNA of interest on its own does not strictly mean that the effect is mediated by the miRNA as one of the only demonstrations would be to remove the miRNA from the miRNA-modified EVs after its production and to see the abrogation of the effect. 


\section{Discussion}

EVs are secreted by various cells that can exhibit a protective or destructive effect on target tissues by transferring their cargo in diverse physiological processes. It has been frequently reported that EVs can modulate cartilage degeneration and regeneration [25-28]. However, these studies have exclusively focused on the role of EVs on cartilage, despite the significance of the adjacent subchondral bone in the context of osteoarthritis [24]. Experimental evidence showed the mechanosensitive nature of miR-221 [16,29,30] and a paracrine effect from chondrocyte to chondrocyte [8] acting mainly on proliferation inside the same tissue. In this study, we demonstrate that chondrocyte derived EVs can inhibit the function of a completely different cell type (osteoblast) in a different tissue (bone), thereby establishing trans-tissue communication.

To date, multiple protocols have been compared for EVs isolation. Differential ultracentrifugation (UC) is acknowledged as the "gold standard", despite the cumbersome centrifugal process and the limitation of mass production [31]. In the present study, the size of vesicles isolated by UC was found to be in a range of 30-200 nm (Figure 1a-d) and the characteristic markers of EVs (Alix, TSG101 and CD81) were identified by Western blot analysis (Figure 1f). This is consistent with current reports, which describe EVs as up to $200 \mathrm{~nm}$ in size vesicles with the above mentioned marker proteins [32-35]. Furthermore, TEM experiments (Figure 1c) confirmed typical morphological EV characteristics, which were consistent with the MISEV2018 guidelines [36].

EV transfer of miRNA has been extensively researched during the past decade. In particular, MSCs isolated from diverse sources (bone marrow, synovial membrane, adipose tissue, umbilical cord and embryonic cells) were reported to be able to transduce miRNA effects through secretion of EVs [37-39], mainly through leading to immunomodulation. Here, we demonstrated that chondrocytes also secrete miRNA through EVs and that the content of the EVs can be controlled by the expression of the miRNA. Similarly, Wang et al. reported that EVs containing miR-221-3p might attenuate OA by enhancing proliferation and migration of cartilage chondrocytes, but the exact mechanism was not further explored [27]. Considering the substantial mechanical/biochemical crosstalk between articular cartilage and subchondral bone $[21,23,24]$, here, we studied EVs as a possible means for cell-cell communication between these tissues. In this regard, miR-221-3p was particularly interesting due to its mechanosensitive nature [16].

Generally, miRNAs can participate in different regulation mechanisms by inhibiting diverse targets, and our results partially confirmed that miRNA-221-3p targets CDKN1B/p27, TIMP-3, Tcf712/TCF4, and ARNT (Figure 2), which are all important cell cycle regulators. In brief, $\mathrm{CDKN} 1 \mathrm{~B} / \mathrm{p} 27$ has been reported to be a versatile regulator of cell proliferation [40]; TIMP-3 can affect bone remodeling as an inhibitor of the matrix metalloproteinases [41]; Tcf712/TCF4 is an important component of the Wnt signaling pathway [42]; and ARNT-also known as a hypoxia-inducible factor (HIF)- $1 \beta$ - was reported to modulate the hypoxia-inducible factor (HIF) pathway [43]. In particular, targets of miR-221-3p were reported to participate in different biological processes and signaling pathways in the cancer field [44], e.g., in the Wnt signaling pathway, which has been also reported to participate in bone management by promoting osteoblast-relevant bone formation and inhibiting osteoclast-relevant bone resorption [45,46]. We found that the transfection of osteoblasts with miR-221-3p mimic led to significant overexpression of miR-221-3p, which consequently inhibited the osteogenic capacity of osteoblasts. This is consistent with another report that showed miR-221 overexpression could significantly decrease the mRNA expression levels of key osteoblast markers (after $24 \mathrm{~h}$ of treatment) in C2C12 cells by targeting RUNX2 [47]. Nevertheless, the long-term outcome of this inhibitory effect has never been shown.

Preceding results showed EVs secreted by chondrocytes contained miR-221-3p, the expression level was consistent with the maternal cells [48] and it changed with mechanical loading $[16,30]$. To simulate the interface between cartilage and bone, and to evaluate how the miR-221-3p secreted by chondrocytes can potentially affect the bone formation 
capacity of osteoblasts, we cocultured these two cells in an in vitro model and found that the signal of overexpressed miR-221-3p in chondrocytes was transferred to osteoblasts with inhibited osteogenic markers expression of COL1A1 and RUNX2 (Figure 6a-b), while the OCN (Figure 6c), a late marker for bone formation [49], was not inhibited. Since direct transfection of osteoblasts with miRNA-221-3p also decreased the OCN expression (Figure 3h), the unchanged expression of the late marker OCN after 2 weeks may be due to slower kinetics of the coculture.

To further clarify the effect of miRNA-221-3p-loaded EVs on osteoblasts, we illustrated that isolated EVs derived from the chondrocytes pre-transfected with miR-221-3p mimic could inhibit the bone formation capacity of osteoblasts both after a short time point of $48 \mathrm{~h}$ (Figure 7), as well as a two-week treatment (Figure 8). After $48 \mathrm{~h}$ treatment with isolated miRNA221-3p-loaded EVs, all miR-221-3p targets were reduced except for the late osteogenic marker OCN. After the longer treatment time of two weeks, the late osteogenic marker OCN was also significantly inhibited, while the expression of early osteogenic markers COL1A1 and RUNX2 returned to control values (Figure 8). This is consistent with Col1A1 and RUNX2, instead being early osteogenic differentiation markers [50,51]. Alizarin Red staining supported the inhibition of osteogenic differentiation by miR221loaded chondrogenic EVs.

Based on our results, we hypothesize that the bone formation capacity of osteoblasts is regulated by the signal of miR-221-3p, mediated by EVs derived from chondrocytes. In previous studies, our group has demonstrated the existence of microchannel structures at the interface of bone and cartilage, which can potentially be pathways for the transportation of EVs and chondrocyte-osteoblast communication [22,23].

Figure 9 provides a schematic representation of how chondrocyte-secreted EVs that contain miR-221-3p may affect osteoblasts via the subchondral bone microchannel network. This could have great implications in understanding the bone-cartilage interface, since miRNAs mediated by EVs are known to be involved in the regulation of knee joint homeostasis and pathogenesis, while several mechanosensitive miRNAs have been demonstrated to be associated with the structural remodeling of bone and cartilage [16,52]. As miRNAs are protected by the EVs through the lipid bilayer membrane, longer communication routes from the maternal cell to the recipient cell would be conceivable [53]

The early stages of OA are often characterized by bone loss caused by increased bone remodeling, followed by sclerosis of subchondral bone, osteophyte formation, and cartilage damage in end-stage OA [54]. This dynamic change in subchondral bone with the development of OA would be particularly interesting when considering the epigenetic regulation of miRNAs during the process. According to Hecht et al., the expression of miR-221 under mechanical loading was elevated only in healthy chondrocytes, not in OA chondrocytes [16]. Other studies have reported decreased expression of miR-221-3p in OA chondrocytes, which is consistent with our results regarding the IL- $1 \beta$-treated chondrocytes (Figure S3e). In light of our results, in both cases, a physiological inhibitory signal on the bone would be lost or diminished, which could eventually support the development of subchondral sclerosis. Hence, the present study may allow us to explain how the dynamic expression of miR-221-3p in cartilage under mechanic loading could affect the structure modification of subchondral bone.

Another physiological process in the course of OA development and subchondral bone remodeling is angiogenesis. It is suggested that angiogenesis may provide additional channels for cell communication and signaling, which includes EVs [55-57]. In accordance with this notion, we found that vascular endothelial growth factor (VEGF) was upregulated in both chondrocytes and osteoblasts after transfection with a miR-221-3p mimic (Figure S3f-g). Additionally, some reports propose that downstream target TIMP-3 is not only involved in the regulation of the matrix metalloproteinases but also is related to angiogenesis during the invasion and metastasis of cancer cells and the protection of myocardial infarction [58,59]. Recently, Zhao et al. reported the TIMP3/TGF- $\beta 1$ axis may be responsi- 
ble for the deterioration and angiogenesis of chondrocytes under mechanical loading [60], which may be related to the regulatory role of miR-221-3p as well in this context.

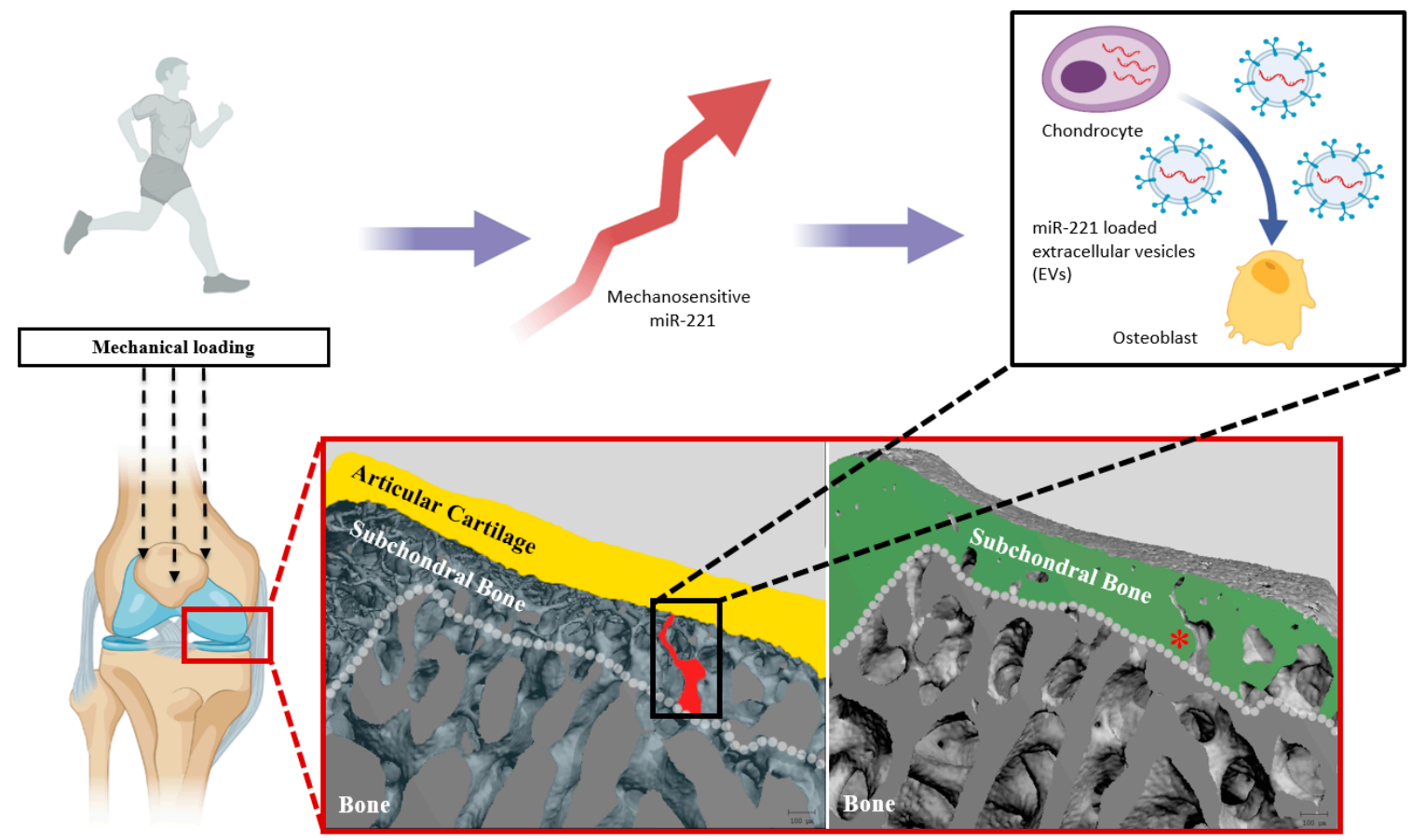

Figure 9. Theoretical scheme of how chondrocyte-secreted EVs harboring miR-221-3p may affect osteoblasts in the physiological condition of joint. The healthy knee joint can be affected by mechanical loading, which increases miR-221-3p expression in the cartilage chondrocytes. Chondrocytes secrete EVs that contain these miRNAs, which can then be taken up by osteoblasts via the microchannel network of the subchondral bone. Subchondral bone (green) and its intricate microarchitecture (red asterisk) are illustrated by the 3D-reconstructed images acquired by high-resolution micro-computed tomography. In the inverted model, the articular cartilage is demonstrated in yellow, while the continuous nature of porous structures (i.e., microchannels; one of which is marked in red color) is visible throughout the entire subchondral bone. These microchannels may be the main pathways of bone-cartilage communication.

An interesting observation was the non-linear effect of miR-221-3p enrichment on the inhibition of targets., i.e., 2725-fold overexpression of miR-221-3p led to a $50 \%$ reduction in target values compared to a $30 \%$ reduction in targets when a 2 -fold miRNA enrichment was observed.

A possible explanation for this can be the sustained action by the miR-221-3p-loaded EVs over $48 \mathrm{~h}$ from the transfected chondrocytes. This might also be indirect evidence for a protective function of the lipid membrane on the miRNA degradation, compared to miRNA alone in cell-cell communication [53]. Zheng et al. have reported that a 3-fold increase in miR-221 in chondrocytes could significantly inhibit its target [13], which is in line with our observation. Murray et al. observed a significant overexpression ( 25,000 fold $)$ of miR-200b via miRNA200b mimics, while the target (Oxr1) expression was reduced by $~ 50 \%$ [61]. Furthermore, Genz et al. could show a 400-fold overexpression of miR-25 after miRNA transfection, leading to a 20-30\% knockdown of the target genes FKBP14 and Adam-17 [62]. These observations confirm our results and the effects are in a similar range.

Furthermore, two studies have reported the miR-221 expression in articular cartilage under mechanical loading in vitro and in vivo, and the results demonstrated that the dynamic change in miR-221 expression in the articular cartilage was around 2-fold [16,30]. Considering the close proximity of bone and cartilage, it may be reasonable that the dynamic change in miR-221 expression in the osteoblasts induced by the transduction of miRNAs from chondrocytes is in the same vicinity. Likewise, it is well known that the regulatory effects of EV-mediated miRNAs are different from miRNAs alone in OA treat- 
ment [63]. If we extrapolate these findings, mechanosensitive miR-221-3p transported between cartilage and bone by EVs might also play a role in other physiological and pathophysiological processes at the border of cartilage and bone including fracture healing, pseudarthrosis formation, osteophyte development, and bone growth. Nevertheless, despite decades of research, our understanding of the exact mechanism of EVs' biogenesis and secretion, transportation, and uptake is still in its infancy. Thus, further studies on the role of EVs-mediated miRNA regulation in the intercellular interaction are required to take advantage of this nanotechnology.

We must indicate that there are several limitations to this study. Firstly, the results were only generated in an in vitro model, which can only, in part, reflect the environment in vivo. If and how EVs are transferred from chondrocytes to osteoblasts in vivo and the mechanisms of how miR-221-3p transferred by EVs inhibits the bone formation capacity of osteoblasts need further research. Our results demonstrated that miR-221-3p-loaded EVs derived from chondrocytes can inhibit osteoblastic function in vitro. However, additional careful evaluations of this inhibitory effect need to be conducted. In particular, it should be noted that overexpression of miR-221-3p in the parental cells probably leads to additional effects due to activation of several miR targets in these parental cells, which may also result in other changes in the produced EVs. Consequently, the increased frequency of the miRNA does not necessarily guarantee that the observed effect is mediated only by the miRNA. Future approaches should therefore include the deletion of the miR-221 to see the possible abrogation of the effect. The effect of mechanics on miR-221-3p expression was stimulated by mimicking oligonucleotides, which cannot fully simulate the physiological and pathological expression of miRNA. Thus, the magnitude of the effect may be different in vivo. Last but not least, how to isolate truly purified EVs and set the physiologic amount of EVs in animal experiments and further in clinical trials would be a crucial issue to be resolved in future studies [64]. Therefore, additional in vitro and in vivo studies are planned to further investigate the cell-cell communication between chondrocytes and osteoblasts via extracellular vesicles.

\section{Materials and Methods}

\subsection{Cell Isolation and Identification}

Chondrocytes and osteoblasts were, respectively, isolated from the knee joint cartilage and calvarium of 3-day-old Wistar rats (Project number T20.1, Central Animal Experimental Facility, University Medical Center Göttingen, Germany) according to a modified enzyme digestion method [65]. Briefly, the snipped cartilage or calvarium was rinsed in precooled PBS (PAN-Biotech, Aidenbach, Germany), digested for $30 \mathrm{~min}$ in $0.25 \%$ trypsin (PAN-Biotech, Aidenbach, Germany) in a $37^{\circ} \mathrm{C}$ water bath, followed by digestion in $0.1 \%$ collagenase II (Sigma Aldrich, St. Louis, MO, USA) at $37^{\circ} \mathrm{C}$ for $4 \mathrm{~h}$. Subsequently, cell suspension was centrifuged and the resulting pellet was resuspended with DMEM-low glucose (Thermo Fisher Scientific, MA, USA) containing 10\% fetal bovine serum (FBS) and $1 \%$ penicillin/streptomycin antibiotics (PAN-Biotech, Aidenbach, Germany) at $37{ }^{\circ} \mathrm{C}$ with $5 \% \mathrm{CO}_{2}$. The medium and the passage cells were changed twice a week and in the case of $90 \%$ confluency, respectively.

The characteristics of the chondrocytes were identified by immunofluorescence. In brief, sections were firstly incubated overnight with primary collagen II antibody (Abcam, Cambridge, UK, 1:50) at $4{ }^{\circ} \mathrm{C}$, and then, incubated with the HRP-coupled secondary antibody (Abcam, Invitrogen, USA, 1:10.000) for one hour at room temperature. Finally, the sections were visualized under a fluorescence microscope (Leica DMi8, Wetzlar, Germany). To identify the osteoblasts, they were seeded in 24-well plates at a density of $5 \times 10^{4}$ and cultured in osteogenic differentiation medium (ODM, DMEM-Low glucose containing $10 \%$ FCS, $1 \%$ penicillin-streptomycin, $0.2 \mathrm{mM}$ ascorbic acid-2-phosphate and $10 \mathrm{mM} \beta$ glycerophosphate) for 2 weeks, followed by Von Kossa as well as Alizarin Red staining (Figure S1). Meanwhile, the osteoblast markers COL1A1, RUNX2, and OCN and the expression of miR-221-3p in osteoblasts were tested by qRT-PCR (Figure S2). 


\subsection{Establishment of OA Model In Vitro}

To establish an in vitro OA model, IL-1 $\beta$ (Peprotech, Rocky Hill, NJ, USA) was adopted to treat chondrocytes as described previously [65]. Briefly, $5 \times 10^{4}$ chondrocytes were seeded in 24-well plates and treated with $10 \mathrm{ng} / \mathrm{mL}$ IL-1 $\beta$ in the complete medium. After $24 \mathrm{~h}$, the cells were harvested for further analysis.

\subsection{Transfection}

MiR-221-3p mimicking oligonucleotides and scrambled control oligonucleotides (QIAGEN, Hilden, Germany) were transfected into chondrocytes at a ratio of $50 \mathrm{nM} / 5 \times 10^{4}$ cells, using Lipofectamine RNAiMAX Transfection Reagent (Thermo Fisher Scientific, MA, USA) according to the manufacturer's instructions. After $6 \mathrm{~h}$ of transfection, the transfected chondrocytes were washed three times with PBS and changed with medium without containing FBS. After $48 \mathrm{~h}$, the cells were collected for further analysis, while the supernatant was collected to isolate EVs loaded with miR-221-3p or scramble control (see Section 4.5.). Similarly, osteoblasts were transfected with the same protocol every three days and 4 times in total. The transfected osteoblasts were collected for further analysis after two weeks.

\subsection{Coculture of Chondrocytes and Osteoblasts}

To establish a cell-cell communication model in vitro, $5 \times 10^{4}$ osteoblasts were seeded in a transwell with a pore size of $3 \mu \mathrm{m}$ (Merck KGaA, Darmstadt, Germany), and cocultured with $3 \times 10^{5}$ chondrocytes pre-transfected with miR-221-3p mimic and scrambled control in 6-well plates for $48 \mathrm{~h}$. A complete medium containing 10\% FBS free of EVs (ultracentrifuged for $16 \mathrm{~h}$ ) and $1 \% \mathrm{P} / \mathrm{S}$ (2.6 mL for chondrocytes and $1.5 \mathrm{~mL}$ for osteoblasts) was used for the process. The cells and supernatant were harvested for further analysis.

\subsection{EVs Isolation and Identification}

According to the recommendation from ISEV (International Society for Extracellular Vesicles), "extracellular vesicle" (EV) was adopted to name the vesicles in the present study [38]. EVs were isolated from a conditioned supernatant of chondrocytes with the classical ultracentrifuge method $[66,67]$. In brief, chondrocytes were cultured in a complete medium until $80 \%$ confluence. After a PBS wash, cells were cultured in a DMEM-low glucose medium without additives for $48 \mathrm{~h}$. Afterwards, the conditioned medium was collected and centrifuged at $500 \times g$ for $5 \mathrm{~min}$, and at $2000 \times g$ for $20 \mathrm{~min}$, to remove dead cells and clear debris and large vesicles, respectively. Thereafter, $100 \mathrm{~mL}$ supernatant underwent ultracentrifugation in polyallomer tubes $(38.5 \mathrm{~mL}$, Beckman Coulter, Brea) for $35 \mathrm{~min}$ at $36,000 \times g$, followed by $2 \mathrm{~h}$ at 110,000 $\times g$ (Optima XPN-80 Ultracentrifuge, SW32 Ti rotor, Beckman Coulter, Brea). Finally, the EV pellets were dissolved in $100 \mu \mathrm{L}$ PBS and stored at $4{ }^{\circ} \mathrm{C}$ for further experiments.

The NanoSight platform (NanoSight LM10, Malvern 24alytical, Kassel, Germany) was used to measure the size distribution and particle concentration of EVs. A LEO912 transmission electron microscope (Carl Zeiss Microscopy, Oberkochen, Germany) and an On Axis 2k CCD camera (TRS-STAR, Stutensee, Germany) were used to observe the morphology of EVs. The specific markers of EVs were detected by Western blot analysis (Section 4.7.)

\subsection{Target-Gene Prediction and Data Analysis}

Public online websites including Targetscan (Retrieved 31 December 2020, from http: / / www.targetscan.org/vert_72/), miRWalk (Retrieved 31 December 2020, from http: / / zmf. umm.uni-heidelberg.de/apps/zmf/mirwalk2/), and miRDB (Retrieved 31 December 2020, from http:/ / mirdb.org/ ) were used to predict the target gene of miR-221-3p. Afterwards, a Venn diagram (Retrieved 31 December 2020, from http: / / bioinformatics.psb.ugent.be/ webtools/Venn/) was used to compile all the predicted targets. To quantify the calcium deposition of osteoblasts after Alizarin red staining, the publicly available software Image J (Version: $1.53 \mathrm{~m}$ ) was adopted (threshold $=120$ ). 


\subsection{Western Blot Analysis}

Total protein was extracted from chondrocyte samples and EV samples using RIPA buffer $(25 \mathrm{mM}$ Tris-HCl pH 7.6,150 mM NaCl, 1\% NP-40, 1\% Natrium-Deoxycholate, $0,1 \%$ SDS). After washing with precooled PBS, protein concentration was measured with a BCA Protein Assay Kit (Thermo Fisher Scientific, MA, USA). Protein samples were mixed with Laemmli (Bio-Rad, US) with a ratio of 4:1, and then heated for $5 \mathrm{~min}$ at $95{ }^{\circ} \mathrm{C}$. Equal amounts of protein were resolved on $12 \%$ SDS-PAGE gels and transferred to polyvinylidene difluoride (PVDF) membranes (Bio-Rad, CA, USA). Membranes were blocked with $5 \%$ skimmed milk for $1 \mathrm{~h}$ and incubated with the primary antibodies COX-2, $\beta$-actin, CD81, Alix, and Tsg101 overnight at $4{ }^{\circ} \mathrm{C}$ (Table S1). After washing with TBST buffer, PVDF membranes were incubated with HRP coupled secondary antibody (Thermo Fisher, MA, USA, 1:10.000) for $1 \mathrm{~h}$. Subsequently, the rinsed membranes were soaked in ECL chemiluminescence reagent (Bio-Rad, CA, USA), and the blots were exposed with the imaging system ChemiDoc XRS + (Bio-Rad, CA, USA). The results were further analyzed via Image Lab Software (Bio-Rad, CA, USA).

\subsection{RNA Isolation and Real-Time $q R T-P C R$}

Total RNA was extracted using Trizol reagent (Invitrogen, Waltham, MA, USA) according to the manufacturer's instructions. RNA concentration and quality were detected with a DS-11 FX + integrated spectrophotometer (Thermo Fisher Scientific, MA, USA). For quantification analysis of mRNA, $1000 \mathrm{ng}$ RNA was reversely transcribed and amplified, while the gene expression was relative to GAPDH. For miRNA analysis, 10 ng RNA was reversely transcribed and amplified using the miRCURY LNA miRNA Kit (QIAGEN, Hilden, Germany) according to the protocols of the manufacturer, while miRNA expression was relative to U6 small nuclear RNA (snRNA). All the calculations for the relative results adopted the standard $2-\Delta \Delta \mathrm{Ct}$ method.

\subsection{Software and Statistical Analysis}

All data were statistically analyzed using GraphPad Prism 5 Software (GraphPad, CA, USA). The Mann-Whitney U-test was used to assess the difference between two groups. Unless otherwise stated, results were shown as mean values with standard deviations, and statistical differences were considered significant when the $p$ value was $<0.05$. All the experiments were repeated with three biological replicates and three technical replicates each. Figures were partly with BioRender.com (May 2021).

\section{Conclusions}

In conclusion, the present study herein demonstrated that EVs from chondrocytes can transfer the mechanosensitive miR-221-3p to osteoblasts, acting as an intercellular messenger and reducing osteoblastic bone formation in vitro. This facilitates a novel perspective on how soft tissues can transduce mechanical cues to adjacent harder tissues through EVs as molecular messengers.

Supplementary Materials: The following are available online at https://www.mdpi.com/article/10.3 390/ijms222413282/s1.

Author Contributions: Conceptualization, A.F.S. and K.O.B.; investigation, X.S. and K.O.B.; resources, A.F.S. and W.L.; writing-original draft preparation, X.S., K.O.B.; writing—review and editing, X.S., K.O.B., S.T., W.L. and A.F.S.; visualization, X.S., K.O.B. and S.T.; supervision, K.O.B., W.L. and A.F.S.; project administration, A.F.S.; funding acquisition, A.F.S. and W.L. All authors have read and agreed to the published version of the manuscript.

Funding: This work was supported by a scholarship from the China Scholarship Council (No. 202008080030).

Institutional Review Board Statement: Animal samples were provided by Central Animal Experimental Facility, University Medical Center Göttingen, Germany (Project number T20.1). 
Informed Consent Statement: Not applicable.

Acknowledgments: We are grateful to Ramona Castro-Machguth, Andrea Koch and especially Kathrin Hannkefor superb technical assistance.

Conflicts of Interest: The authors declare no conflict of interest.

\section{References}

1. Hügle, T. Update Osteoarthritis. Rev. Med. Suisse 2020, 16, 500-502. [PubMed]

2. Hunt, M.A.; Charlton, J.M.; Esculier, J.-F. Osteoarthritis Year in Review 2019: Mechanics. Osteoarthr. Cartil. 2020, $28,267-274$. [CrossRef] [PubMed]

3. Loeser, R.F.; Goldring, S.R.; Scanzello, C.R.; Goldring, M.B. Osteoarthritis: A Disease of the Joint as an Organ. Arthritis Rheum. 2012, 64, 1697-1707. [CrossRef]

4. Kloppenburg, M.; Berenbaum, F. Osteoarthritis Year in Review 2019: Epidemiology and Therapy. Osteoarthr. Cartil. 2020, 28, 242-248. [CrossRef]

5. Kolasinski, S.L.; Neogi, T.; Hochberg, M.C.; Oatis, C.; Guyatt, G.; Block, J.; Callahan, L.; Copenhaver, C.; Dodge, C.; Felson, D.; et al. 2019 American College of Rheumatology/Arthritis Foundation Guideline for the Management of Osteoarthritis of the Hand, Hip, and Knee. Arthritis Care Res. 2020, 72, 149-162. [CrossRef]

6. Cui, A.; Li, H.; Wang, D.; Zhong, J.; Chen, Y.; Lu, H. Global, Regional Prevalence, Incidence and Risk Factors of Knee Osteoarthritis in Population-Based Studies. EClinicalMedicine 2020, 29, 1-13. [CrossRef]

7. Zhang, W.; Ouyang, H.; Dass, C.R.; Xu, J. Current Research on Pharmacologic and Regenerative Therapies for Osteoarthritis. Bone Res. 2016, 4, 1-14. [CrossRef] [PubMed]

8. Liu, X.; Shortt, C.; Zhang, F.; Bater, M.Q.; Cowman, M.K.; Kirsch, T. Extracellular Vesicles Released From Articular Chondrocytes Play a Major Role in Cell-Cell Communication. J. Orthop. Res. Off. Publ. Orthop. Res. Soc. 2020, 38, 731-739. [CrossRef]

9. Reynard, L.N.; Barter, M.J. Osteoarthritis Year in Review 2019: Genetics, Genomics and Epigenetics. Osteoarthr. Cartil. 2020, 28, 275-284. [CrossRef]

10. Lim, L.P.; Lau, N.C.; Garrett-Engele, P.; Grimson, A.; Schelter, J.M.; Castle, J.; Bartel, D.P.; Linsley, P.S.; Johnson, J.M. Microarray Analysis Shows That Some MicroRNAs Downregulate Large Numbers of Target MRNAs. Nature 2005, 433, 769-773. [CrossRef]

11. Yu, X.-M.; Meng, H.-Y.; Yuan, X.-L.; Wang, Y.; Guo, Q.-Y.; Peng, J.; Wang, A.-Y.; Lu, S.-B. MicroRNAs' Involvement in Osteoarthritis and the Prospects for Treatments. Evid. Based Complement. Alternat. Med. 2015, 2015, 1-13. [CrossRef] [PubMed]

12. Shang, X.; Böker, K.O.; Taheri, S.; Hawellek, T.; Lehmann, W.; Schilling, A.F. The Interaction between MicroRNAs and the Wnt/ $\beta$-Catenin Signaling Pathway in Osteoarthritis. Int. J. Mol. Sci. 2021, 22, 9887. [CrossRef] [PubMed]

13. Zheng, X.; Zhao, F.-C.; Pang, Y.; Li, D.-Y.; Yao, S.-C.; Sun, S.-S.; Guo, K.-J. Downregulation of MiR-221-3p Contributes to IL-1 $\beta$-Induced Cartilage Degradation by Directly Targeting the SDF1/CXCR4 Signaling Pathway. J. Mol. Med. Berl. Ger. 2017, 95, 615-627. [CrossRef] [PubMed]

14. Yang, M.; Zhang, L.; Gibson, G.J. Chondrocyte MiRNAs 221 and 483-5p Respond to Loss of Matrix Interaction by Modulating Proliferation and Matrix Synthesis. Connect. Tissue Res. 2015, 56, 236-243. [CrossRef]

15. Lolli, A.; Narcisi, R.; Lambertini, E.; Penolazzi, L.; Angelozzi, M.; Kops, N.; Gasparini, S.; van Osch, G.J.V.M.; Piva, R. Silencing of Antichondrogenic MicroRNA-221 in Human Mesenchymal Stem Cells Promotes Cartilage Repair In Vivo. Stem Cells Dayt. Ohio 2016, 34, 1801-1811. [CrossRef]

16. Hecht, N.; Johnstone, B.; Angele, P.; Walker, T.; Richter, W. Mechanosensitive MiRs Regulated by Anabolic and Catabolic Loading of Human Cartilage. Osteoarthr. Cartil. 2019, 27, 1208-1218. [CrossRef] [PubMed]

17. Grad, S.; Lee, C.R.; Gorna, K.; Gogolewski, S.; Wimmer, M.A.; Alini, M. Surface Motion Upregulates Superficial Zone Protein and Hyaluronan Production in Chondrocyte-Seeded Three-Dimensional Scaffolds. Tissue Eng. 2005, 11, 249-256. [CrossRef]

18. Musumeci, G. The Effect of Mechanical Loading on Articular Cartilage. J. Funct. Morphol. Kinesiol. 2016, 1, 154. [CrossRef]

19. Lai, Y.-S.; Chen, W.-C.; Huang, C.-H.; Cheng, C.-K.; Chan, K.-K.; Chang, T.-K. The Effect of Graft Strength on Knee Laxity and Graft In-Situ Forces after Posterior Cruciate Ligament Reconstruction. PLoS ONE 2015, 10, e0168280. [CrossRef]

20. Findlay, D.M.; Kuliwaba, J.S. Bone-Cartilage Crosstalk: A Conversation for Understanding Osteoarthritis. Bone Res. 2016, 4, 1-12. [CrossRef]

21. Yuan, X.L.; Meng, H.Y.; Wang, Y.C.; Peng, J.; Guo, Q.Y.; Wang, A.Y.; Lu, S.B. Bone-Cartilage Interface Crosstalk in Osteoarthritis: Potential Pathways and Future Therapeutic Strategies. Osteoarthr. Cartil. 2014, 22, 1077-1089. [CrossRef] [PubMed]

22. Taheri, S.; Winkler, T.; Schenk, L.S.; Neuerburg, C.; Baumbach, S.F.; Zustin, J.; Lehmann, W.; Schilling, A.F. Developmental Transformation and Reduction of Connective Cavities within the Subchondral Bone. Int. J. Mol. Sci. 2019, 20, 770. [CrossRef] [PubMed]

23. Taheri, S.; Yoshida, T.; Böker, K.O.; Foerster, R.H.; Jochim, L.; Flux, A.L.; Grosskopf, B.; Lehmann, W.; Schilling, A.F. Investigating the Microchannel Architectures Inside the Subchondral Bone in Relation to Estimated Hip Reaction Forces on the Human Femoral Head. Calcif. Tissue Int. 2021, 1, 1-15. [CrossRef]

24. Pan, J.; Zhou, X.; Li, W.; Novotny, J.E.; Doty, S.B.; Wang, L. In Situ Measurement of Transport between Subchondral Bone and Articular Cartilage. J. Orthop. Res. 2009, 27, 1347-1352. [CrossRef] 
25. Wang, Y.; Yu, D.; Liu, Z.; Zhou, F.; Dai, J.; Wu, B.; Zhou, J.; Heng, B.C.; Zou, X.H.; Ouyang, H.; et al. Exosomes from Embryonic Mesenchymal Stem Cells Alleviate Osteoarthritis through Balancing Synthesis and Degradation of Cartilage Extracellular Matrix. Stem. Cell Res. Ther. 2017, 8, 1-13. [CrossRef] [PubMed]

26. Kolhe, R.; Hunter, M.; Liu, S.; Jadeja, R.N.; Pundkar, C.; Mondal, A.K.; Mendhe, B.; Drewry, M.; Rojiani, M.V.; Liu, Y.; et al. Gender-Specific Differential Expression of Exosomal MiRNA in Synovial Fluid of Patients with Osteoarthritis. Sci. Rep. 2017, 7, 2029. [CrossRef] [PubMed]

27. Mao, G.; Hu, S.; Zhang, Z.; Wu, P.; Zhao, X.; Lin, R.; Liao, W.; Kang, Y. Exosomal MiR-95-5p Regulates Chondrogenesis and Cartilage Degradation via Histone Deacetylase 2/8. J. Cell. Mol. Med. 2018, 22, 5354-5366. [CrossRef]

28. Wang, R.; Jiang, W.; Zhang, L.; Xie, S.; Zhang, S.; Yuan, S.; Jin, Y.; Zhou, G. Intra-Articular Delivery of Extracellular Vesicles Secreted by Chondrogenic Progenitor Cells from MRL/MpJ Superhealer Mice Enhances Articular Cartilage Repair in a Mouse Injury Model. Stem Cell Res. Ther. 2020, 11, 1-14. [CrossRef]

29. Dunn, W.; DuRaine, G.; Reddi, A.H. Profiling MicroRNA Expression in Bovine Articular Cartilage and Implications for Mechanotransduction. Arthritis Rheum. 2009, 60, 2333-2339. [CrossRef]

30. Stadnik, P.S.; Gilbert, S.J.; Tarn, J.; Charlton, S.; Skelton, A.J.; Barter, M.J.; Duance, V.C.; Young, D.A.; Blain, E.J. Regulation of MicroRNA-221, -222, -21 and -27 in Articular Cartilage Subjected to Abnormal Compressive Forces. J. Physiol. 2021, 599, 143-155. [CrossRef]

31. Brennan, K.; Martin, K.; FitzGerald, S.P.; O'Sullivan, J.; Wu, Y.; Blanco, A.; Richardson, C.; Gee, M.M.M. A Comparison of Methods for the Isolation and Separation of Extracellular Vesicles from Protein and Lipid Particles in Human Serum. Sci. Rep. 2020, 10, 1039. [CrossRef] [PubMed]

32. Nakase, I.; Noguchi, K.; Fujii, I.; Futaki, S. Vectorization of Biomacromolecules into Cells Using Extracellular Vesicles with Enhanced Internalization Induced by Macropinocytosis. Sci. Rep. 2016, 6, 34937. [CrossRef] [PubMed]

33. Eguchi, T.; Sogawa, C.; Ono, K.; Matsumoto, M.; Tran, M.T.; Okusha, Y.; Lang, B.J.; Okamoto, K.; Calderwood, S.K. Cell Stress Induced Stressome Release Including Damaged Membrane Vesicles and Extracellular HSP90 by Prostate Cancer Cells. Cells 2020, 9, 755. [CrossRef]

34. Liang, L.-G.; Kong, M.-Q.; Zhou, S.; Sheng, Y.-F.; Wang, P.; Yu, T.; Inci, F.; Kuo, W.P.; Li, L.-J.; Demirci, U.; et al. An Integrated Double-Filtration Microfluidic Device for Isolation, Enrichment and Quantification of Urinary Extracellular Vesicles for Detection of Bladder Cancer. Sci. Rep. 2017, 7, 46224. [CrossRef]

35. Pérez-González, R.; Gauthier, S.A.; Sharma, A.; Miller, C.; Pawlik, M.; Kaur, G.; Kim, Y.; Levy, E. A Pleiotropic Role for Exosomes Loaded with the Amyloid $\beta$ Precursor Protein Carboxyl-Terminal Fragments in the Brain of Down Syndrome Patients. Neurobiol. Aging 2019, 84, 26-32. [CrossRef] [PubMed]

36. Théry, C.; Witwer, K.W.; Aikawa, E.; Alcaraz, M.J.; Anderson, J.D.; Andriantsitohaina, R.; Antoniou, A.; Arab, T.; Archer, F.; Atkin-Smith, G.K.; et al. Minimal Information for Studies of Extracellular Vesicles 2018 (MISEV2018): A Position Statement of the International Society for Extracellular Vesicles and Update of the MISEV2014 Guidelines. J. Extracell. Vesicles 2018, 7, 1-47. [CrossRef]

37. Mohd Noor, N.A.; Abdullah Nurul, A.; Ahmad Mohd Zain, M.R.; Wan Nor Aduni, W.K.; Azlan, M. Extracellular Vesicles from Mesenchymal Stem Cells as Potential Treatments for Osteoarthritis. Cells 2021, 10, 1287. [CrossRef]

38. Tan, S.S.H.; Tjio, C.K.E.; Wong, J.R.Y.; Wong, K.L.; Chew, J.R.J.; Hui, J.H.P.; Toh, W.S. Mesenchymal Stem Cell Exosomes for Cartilage Regeneration: A Systematic Review of Preclinical In Vivo Studies. Tissue Eng. Part B Rev. 2021, 27, 1-13. [CrossRef] [PubMed]

39. Taghiyar, L.; Jahangir, S.; Khozaei Ravari, M.; Shamekhi, M.A.; Eslaminejad, M.B. Cartilage Repair by Mesenchymal Stem Cell-Derived Exosomes: Preclinical and Clinical Trial Update and Perspectives. Adv. Exp. Med. Biol. 2021, 12, 73-93. [CrossRef]

40. Hu, X.-H.; Zhao, Z.-X.; Dai, J.; Geng, D.-C.; Xu, Y.-Z. MicroRNA-221 Regulates Osteosarcoma Cell Proliferation, Apoptosis, Migration, and Invasion by Targeting CDKN1B/P27. J. Cell. Biochem. 2019, 120, 4665-4674. [CrossRef]

41. Poulet, B.; Liu, K.; Plumb, D.; Vo, P.; Shah, M.; Staines, K.; Sampson, A.; Nakamura, H.; Nagase, H.; Carriero, A.; et al. Overexpression of TIMP-3 in Chondrocytes Produces Transient Reduction in Growth Plate Length but Permanently Reduces Adult Bone Quality and Quantity. PLoS ONE 2016, 11, e0167971. [CrossRef] [PubMed]

42. Wang, Y.; Fan, X.; Xing, L.; Tian, F. Wnt Signaling: A Promising Target for Osteoarthritis Therapy. Cell Commun. Signal. CCS 2019, 17, 1-14. [CrossRef]

43. Liang, C.; Li, J.; Lu, C.; Xie, D.; Liu, J.; Zhong, C.; Wu, X.; Dai, R.; Zhang, H.; Guan, D.; et al. HIF1 $\alpha$ Inhibition Facilitates Leflunomide-AHR-CRP Signaling to Attenuate Bone Erosion in CRP-Aberrant Rheumatoid Arthritis. Nat. Commun. 2019, 10, 1-13. [CrossRef]

44. Garofalo, M.; Quintavalle, C.; Romano, G.; Croce, C.M.; Condorelli, G. MiR221/222 in Cancer: Their Role in Tumor Progression and Response to Therapy. Curr. Mol. Med. 2012, 12, 27-33. [CrossRef]

45. Glass, D.A.; Bialek, P.; Ahn, J.D.; Starbuck, M.; Patel, M.S.; Clevers, H.; Taketo, M.M.; Long, F.; McMahon, A.P.; Lang, R.A.; et al. Canonical Wnt Signaling in Differentiated Osteoblasts Controls Osteoclast Differentiation. Dev. Cell 2005, 8, 751-764. [CrossRef]

46. Weivoda, M.M.; Ruan, M.; Hachfeld, C.M.; Pederson, L.; Howe, A.; Davey, R.A.; Zajac, J.D.; Kobayashi, Y.; Williams, B.O.; Westendorf, J.J.; et al. Wnt Signaling Inhibits Osteoclast Differentiation by Activating Canonical and Noncanonical CAMP/PKA Pathways. J. Bone Miner. Res. Off. J. Am. Soc. Bone Miner. Res. 2016, 31, 65-75. [CrossRef] 
47. Zhang, Y.; Gao, Y.; Cai, L.; Li, F.; Lou, Y.; Xu, N.; Kang, Y.; Yang, H. MicroRNA-221 Is Involved in the Regulation of Osteoporosis through Regulates RUNX2 Protein Expression and Osteoblast Differentiation. Am. J. Transl. Res. 2017, 9, $126-135$.

48. Zhang, Y.; Liu, Y.; Liu, H.; Tang, W.H. Exosomes: Biogenesis, Biologic Function and Clinical Potential. Cell Biosci. 2019, 9, 19. [CrossRef] [PubMed]

49. Boskey, A.L.; Wians, F.H.; Hauschka, P.V. The Effect of Osteocalcin on in Vitro Lipid-Induced Hydroxyapatite Formation and Seeded Hydroxyapatite Growth. Calcif. Tissue Int. 1985, 37, 57-62. [CrossRef] [PubMed]

50. Kannan, S.; Ghosh, J.; Dhara, S.K. Osteogenic Differentiation Potential of Porcine Bone Marrow Mesenchymal Stem Cell Subpopulations Selected in Different Basal Media. Biol. Open 2020, 9, 1-11. [CrossRef]

51. Qin, X.; Jiang, Q.; Komori, H.; Sakane, C.; Fukuyama, R.; Matsuo, Y.; Ito, K.; Miyazaki, T.; Komori, T. Runt-Related Transcription Factor-2 (Runx2) Is Required for Bone Matrix Protein Gene Expression in Committed Osteoblasts in Mice. J. Bone Miner. Res. Off. J. Am. Soc. Bone Miner. Res. 2021, 36, 1-15. [CrossRef]

52. Chen, Z.; Zhang, Y.; Liang, C.; Chen, L.; Zhang, G.; Qian, A. Mechanosensitive MiRNAs and Bone Formation. Int. J. Mol. Sci. 2017, 18, 1684. [CrossRef]

53. Kuang, Y.; Zheng, X.; Zhang, L.; Ai, X.; Venkataramani, V.; Kilic, E.; Hermann, D.M.; Majid, A.; Bähr, M.; Doeppner, T.R. AdiposeDerived Mesenchymal Stem Cells Reduce Autophagy in Stroke Mice by Extracellular Vesicle Transfer of MiR-25. J. Extracell. Vesicles 2020, 10, 1-20. [CrossRef]

54. Burr, D.B.; Gallant, M.A. Bone Remodelling in Osteoarthritis. Nat. Rev. Rheumatol. 2012, 8, 665-673. [CrossRef]

55. Goldring, M.B.; Goldring, S.R. Osteoarthritis. J. Cell. Physiol. 2007, 213, 626-634. [CrossRef]

56. Stucker, S.; Chen, J.; Watt, F.E.; Kusumbe, A.P. Bone Angiogenesis and Vascular Niche Remodeling in Stress, Aging, and Diseases. Front. Cell Dev. Biol. 2020, 8, 1-20. [CrossRef]

57. Bouland, C.; Philippart, P.; Dequanter, D.; Corrillon, F.; Loeb, I.; Bron, D.; Lagneaux, L.; Meuleman, N. Cross-Talk Between Mesenchymal Stromal Cells (MSCs) and Endothelial Progenitor Cells (EPCs) in Bone Regeneration. Front. Cell Dev. Biol. 2021, 9, 1-17. [CrossRef] [PubMed]

58. Takawale, A.; Zhang, P.; Azad, A.; Wang, W.; Wang, X.; Murray, A.G.; Kassiri, Z. Myocardial Overexpression of TIMP3 after Myocardial Infarction Exerts Beneficial Effects by Promoting Angiogenesis and Suppressing Early Proteolysis. Am. J. Physiol. Heart Circ. Physiol. 2017, 313, H224-H236. [CrossRef] [PubMed]

59. Huang, H.-L.; Liu, Y.-M.; Sung, T.-Y.; Huang, T.-C.; Cheng, Y.-W.; Liou, J.-P.; Pan, S.-L. TIMP3 Expression Associates with Prognosis in Colorectal Cancer and Its Novel Arylsulfonamide Inducer, MPT0B390, Inhibits Tumor Growth, Metastasis and Angiogenesis. Theranostics 2019, 9, 6676-6689. [CrossRef] [PubMed]

60. Zhao, D.-L.; Li, H.-T.; Liu, S.-H. TIMP3/TGF- $\beta 1$ Axis Regulates Mechanical Loading-induced Chondrocyte Degeneration and Angiogenesis. Mol. Med. Rep. 2020, 22, 2637-2644. [CrossRef] [PubMed]

61. Murray, A.R.; Chen, Q.; Takahashi, Y.; Zhou, K.K.; Park, K.; Ma, J. MicroRNA-200b Downregulates Oxidation Resistance 1 (Oxr1) Expression in the Retina of Type 1 Diabetes Model. Investig. Ophthalmol. Vis. Sci. 2013, 54, 1689-1697. [CrossRef] [PubMed]

62. Genz, B.; Coleman, M.A.; Irvine, K.M.; Kutasovic, J.R.; Miranda, M.; Gratte, F.D.; Tirnitz-Parker, J.E.E.; Olynyk, J.K.; Calvopina, D.A.; Weis, A.; et al. Overexpression of MiRNA-25-3p Inhibits Notch1 Signaling and TGF- $\beta$-Induced Collagen Expression in Hepatic Stellate Cells. Sci. Rep. 2019, 9, 8541. [CrossRef] [PubMed]

63. Esmaeili, A.; Hosseini, S.; Baghaban Eslaminejad, M. Engineered-Extracellular Vesicles as an Optimistic Tool for MicroRNA Delivery for Osteoarthritis Treatment. Cell. Mol. Life Sci. CMLS 2021, 78, 79-91. [CrossRef]

64. Webber, J.; Clayton, A. How Pure Are Your Vesicles? J. Extracell. Vesicles 2013, 2, 823-831. [CrossRef]

65. Wang, P.; Mao, Z.; Pan, Q.; Lu, R.; Huang, X.; Shang, X.; Zhang, R.; You, H. Histone Deacetylase-4 and Histone Deacetylase-8 Regulate Interleukin-1 $\beta$-Induced Cartilage Catabolic Degradation through MAPK/JNK and ERK Pathways. Int. J. Mol. Med. 2018, 41, 2117-2127. [CrossRef] [PubMed]

66. Momen-Heravi, F. Isolation of Extracellular Vesicles by Ultracentrifugation. Methods Mol. Biol. Clifton NJ 2017, 1660, 25-32. [CrossRef]

67. Konoshenko, M.Y.; Lekchnov, E.A.; Vlassov, A.V.; Laktionov, P.P. Isolation of Extracellular Vesicles: General Methodologies and Latest Trends. BioMed Res. Int. 2018, 2018, 1-27. [CrossRef] 